\title{
ON CONTINUITY AND OTHER ANALYTIC PROPERTIES OF STOCHASTIC PROCESS SAMPLE FUNCTIONS ${ }^{1}$
}

M. R. LEADBETTER AND EDWARD W. WEISSNER

1. Introduction. Certain general results concerning continuity and other analytic properties of the sample functions of a stochastic process $\xi(t)$, involve assumptions of the type

$$
\operatorname{Pr}\{|\xi(t+h)-\xi(t)| \geqq g(h)\} \leqq q(h),
$$

where $g(h)$ and $q(h)$ are functions with particular properties near $h=0$. For example if

$$
g(h), q(h) \text { are even, nonincreasing as } h \downarrow 0,
$$

and

$$
\sum_{n=0}^{\infty} g\left(2^{-n}\right)<\infty, \quad \sum_{n=0}^{\infty} 2^{n} q\left(2^{-n}\right)<\infty
$$

then it follows (cf. [4], [1]) that there is a process $\eta(t)$, equivalent to $\xi(t)$ and, with probability one, possessing everywhere continuous sample functions. (Equivalence of $\xi$ and $\eta$ has the usual meaning that, for each fixed $t, \xi(t)=\eta(t)$ with probability one.)

A number of interesting results follow as corollaries from this theorem. For example if $\xi(t)$ is a (zero mean) normal stochastic process satisfying

$$
\varepsilon|\xi(t+h)-\xi(t)|^{2}<C /|\log | h||^{a}
$$

for some $C>0, a>3$, and all sufficiently small $h$, then $g$ and $q$ can be chosen so that (2) and (3) are satisfied and hence (an equivalent version of) $\xi(t)$ has, with probability one, continuous sample functions. However it is known (cf. [3], [2]) that if $\xi(t)$ is a normal process, the condition (4) may be weakened (e.g. by requiring only $a>1$ ) and still give a sufficient condition for continuity. This latter result does not appear to follow from the general theorem quoted above.

In this note we shall give a general result for sample function continuity from which, in particular, the more delicate results of [3] for normal processes will follow. This result is obtained by modifying the form of condition (3). The derivation uses methods very similar to those in [4], [1] and especially [2], and will be sketched only. In

Received by the editors August 12, 1968.

1 Research supported by the Office of Naval Research, Contract Nonr 855(09). 
addition we state corresponding theorems for the existence of a continuous sample derivative from which some results given in [4] and [1] follow as particular cases. The results given, apply to processes defined on the real line. While this is the most interesting case, we note that it is possible to adapt some of the results to apply in more abstract settings.

2. Continuity of sample functions. Consider a real (or complex) stochastic process $\xi(t)$ defined on $0 \leqq t \leqq 1$. The following easily proved lemma enables us to replace $[0,1]$ by a countable dense subset in discussing continuity.

Leмma. Given $\xi(t)$, there is an equivalent process $\{\eta(t): 0 \leqq t \leqq 1\}$ whose sample functions are, with probability one, continuous on $[0,1]$, if and only if

(a) $\xi(t)$ is continuous in probability on $[0,1]$, and

(b) there is a countable dense subset $D$ of $[0,1]$ on which $\xi(t)$ is uniformly continuous with probability one.

Using this lemma we may obtain the main continuity theorem. In the statement of this and later results, $r_{1}, r_{2}, \cdots$ will denote an arbitrary sequence of integers, each greater than one, and $h_{n}$ $=\left(r_{1} r_{2} \cdots r_{n}\right)^{-1}$.

Theorem 1. Suppose that $\operatorname{Pr}\{|\xi(t+h)-\xi(t)| \geqq g(h)\} \leqq q(h)$ for all $t$, $t+h$ in $[0,1]$ where $g, q$ are even functions of $h, q(h)$ being nonincreasing as $h \downarrow 0$ and where

(a) $\sum_{1}^{\infty} g\left(h_{n}\right)<\infty, \sum_{1}^{\infty} h_{n+1}^{-1} q\left(h_{n}\right)<\infty$,

(b) $g(h) \rightarrow 0$ as $h \rightarrow 0$ and there is a constant $K$ such that $g\left(s h_{n+1}\right)$ $\leqq K g\left(h_{n}\right)$ for $s=1,2 \cdots r_{n+1}, n=1,2 \cdots$.

Then there exists a process $\eta(t)$, equivalent to $\xi(t)$, and possessing, with probability one, continuous sample functions on $[0,1]$.

Sketch of Proof. Since for $h$ sufficiently small

$$
\begin{aligned}
\operatorname{Pr}\{|\xi(t+h)-\xi(t)| & \geqq \epsilon\} \leqq \operatorname{Pr}\{|\xi(t+h)-\xi(t)| \geqq g(h)\} \\
& \leqq q(h) \rightarrow 0,
\end{aligned}
$$

it follows that $\xi$ is continuous in probability. Hence it only remains to show uniform continuity on a countable dense subset of $[0,1]$.

Write $t_{n, 8}=s h_{n}$ and $D=\left\{t_{n, 8}: s=0,1, \cdots h_{n}^{-1}, n=1,2 \cdots\right\} . D$ is clearly a countable dense subset of $[0,1]$. Now using (b) and the monotonicity of $q$, it is easily seen that

$\operatorname{Pr}\left\{\max _{0 \leq r<h_{n}^{-1}} \max _{0<s \leq r_{n+1}}\left|\xi\left(r h_{n}+s h_{n+1}\right)-\xi\left(r h_{n}\right)\right| \geqq K g\left(h_{n}\right\} \leqq h_{n+1}^{-1} q\left(h_{n}\right)\right.$ 
and since $\sum h_{n+1}^{-1} q\left(h_{n}\right)<\infty$, the Borel-Cantelli Lemma shows that there is a random variable $N$ such that

$$
\left|\xi\left(r h_{n}+s h_{n+1}\right)-\xi\left(r h_{n}\right)\right| \leqq K g\left(h_{n}\right)
$$

for any integers $n, r, s$ such that $n \geqq N, 0 \leqq r<h_{n}^{-1}, 0 \leqq s \leqq r_{n+1}$.

Given $\epsilon>0$ let $M$ be such that $\sum_{M}^{\infty} g\left(h_{n}\right)<\epsilon /(3 K)$ and write $N_{0}$ for the random variable $N+M+1$. Using (5) it may be shown that if $s_{1}, s_{2} \in D$ and $\left|s_{1}-s_{2}\right|<h_{N_{0}}$, then

$$
\left|\xi\left(s_{1}\right) .-\xi\left(s_{2}\right)\right| \leqq K\left[2 \sum_{j=N_{0}}^{\infty} g\left(h_{j}\right)+g\left(h_{N_{0}}\right)\right]<\epsilon
$$

from which the required uniform continuity on $D$ follows.

COROLLARY. In particular the theorem remains true if condition (b) is replaced by the simpler requirement that $g(h)$ be nonincreasing as $h \downarrow 0$.

Application to normal processes. The weakest sufficient condition we are aware of for sample function continuity in the normal case is the following, given by Fernique [3]. Suppose that $\xi(t)$ is a (zero mean) normal process for which

$$
\varepsilon|\xi(t+h)-\xi(t)|^{2} \leqq \psi^{2}(h) \quad \text { all } t, t+h \in[0,1],
$$

where $\psi$ is even and nonincreasing as $h \downarrow 0$. If $\psi$ satisfies the further condition $\int^{\infty} \psi\left(e^{-x^{2}}\right) d x<\infty$, then $\xi$ is equivalent to a process $\eta(t)$ possessing, with probability one, continuous sample functions on $[0,1]$. This result may be obtained as an application of Theorem 1 , and we now briefly indicate the details.

Specifically, suppose $\xi(t)$ is a zero mean normal process satisfying (6) with $\int^{\infty} \psi\left(\left[\exp -x^{2}\right]\right) d x<\infty$. Write $h_{n}=2^{-2^{n}}$ and $g(h)$ $=4|\log | h|| 1 / 2 \psi(h), \quad q(h)=\frac{1}{4}|\log | h||^{-1 / 2} e^{-8|\log | h||}$. Then it follows from the integrability assumption on $\psi$ that $\sum g\left(h_{n}\right)<\infty$. The remaining assumptions of Theorem 1 are easily checked.

Finally we note that the result mentioned in $\$ 1$ involving equation (4) with $a>1$ can be deduced from the above result of Fernique, or directly from Theorem 1 (again with $h_{n}=2^{-2^{n}}$ ).

3. Other analytic properties. One may obtain similar results to Theorem 1, using the same methods, for differentiability of sample functions of a stochastic process $\xi(t)$, or for the sample functions to satisfy Lipschitz conditions. As examples, the following two results yield sufficient conditions for the existence of a continuous sample derivative. (These include certain results of [4] and [1].) In the statements of these theorems, $r_{n}$ and $h_{n}$ are as previously defined.

Theorem 2. Suppose $\operatorname{Pr}\{|\xi(t+h)-\xi(t)| \geqq g(h)\} \leqq q(h)$ for all $t$, 
$t+h \in[0,1]$ where $g$ and $q$ are even functions of $h$, nonincreasing as $h \downarrow 0$. Suppose that $\sum h_{n}^{-1} g\left(h_{n}\right)<\infty$ and $\sum h_{n}^{-1} q\left(h_{n}\right)<\infty$. Then $\xi(t)$ is equivalent to a process $\eta(t)$ which, with probability one, has a continuous sample derivative on $[0,1]$.

ThEOREM 3. Suppose $\xi(t)$ is continuous in probability on $[0,1]$ and that

$$
\operatorname{Pr}\{|\xi(t+h)-2 \xi(t)+\xi(t-h)| \geqq g(h)\} \leqq q(h)
$$

for all $t, t+h, t-h \in[0,1]$ where $g$ and $q$ are even, nonincreasing as $h \downarrow 0$, and satisfy

$$
\sum h_{n}^{-1} r_{n} g\left(h_{n}\right)<\infty, \quad \sum h_{n}^{-1} q\left(h_{n}\right)<\infty .
$$

Then again $\xi$ is equivalent to a process $\eta(t)$ which has, with probability one, a continuous derivative on $[0,1]$.

Theorems 2 and 3 contain different conditions on the process $\xi(t)$. The conditions of Theorem 3 involving $\xi(t+h), \xi(t), \xi(t-h)$ are weaker, in some cases, than those of Theorem 2. The conditions of Theorem 2 are, however, likely to be easier to apply in general.

\section{REFERENCES}

1. Harald Cramér and M. R. Leadbetter, Stationary and related stochastic processes, Wiley, New York, 1967.

2. R. M. Dudley, Gaussian processes on several parameters, Ann. Math. Statist. 36 (1965), 771-787.

3. X. Fernique, Continuite des processus Gaussiens, C. R. Acad. Sci. Paris 259 (1964), 6058-6060.

4. M. Loève, Probability theory, Van Nostrand, Princeton, N. J., 1960.

University of North Carolina 